\title{
Althusser et la lecture des classiques de la pensée politique moderne: Notes pour une recherche sur sa pratique
}

\author{
Althusser and his reading of modern classical \\ political thought: Notes towards an \\ investigation on his practice
}

MARCO RAMPAZZO BAZZAN ${ }^{a}$

\section{Résumé}

Cet article vise à élucider le protocole de lecture que Louis Althusser forge pour son interprétation des classiques de la pensée politique moderne (Hobbes, Rousseau, Montesquieu). À l'appui des critiques formulées à ce sujet par Jacques Rancière, ce travail présente une hypothèse de recherche sur la manière dans laquelle Althusser développa son étude de l'histoire de la philosophie politique dans ses cours et écrits. Son but est de questionner ce qu'Étienne Balibar définit comme la singularité d'Althusser, à savoir sa double appartenance : militant du Parti Communiste Français d'un côté ; et maîtreassistant à l'École Normale Supérieure de l'autre. Sous cette optique l'article explore stratégies et contradictions développées par Althusser au fil de sa lecture des auteurs du $18^{\text {ème }}$ siècle. Il tâche de répondre à des questions telles que : Comment organise-t-il son étude de la philosophie politique

a Universidade Federal do Espírito Santo (UFES), Espírito Santo, ES, Brasil. Doutor em Filosofia Política e História do pensamento político em Filosofia, e-mail: marco.rampazzo.bazzan@gmail.com 
moderne comme " une propédeutique à l'intelligence de Marx » ?; Quels sont les problèmes 'imposés par la conjoncture' révisionniste qui orientent son travail sur la philosophie à l'âge classique ? ; Comment son protocole dérive de ses présupposés marxiens et leniniens, tels qu'il les élabore dans le Pour Marx et dans I'Introduction à Lire le Capital ? Ainsi, la finalité de l'article est de montrer comme ces présupposés vont également constituer les 'obstacles épistémologiques', à savoir les limites de sa compréhension de la philosophie politique moderne. Cela à partir de sa thèse majeure, c'est-à-dire l'existence d'une coupure épistémologique dans l'œuvre de Marx.

Mots-clés : Louis Althusser. Jacques Rancière. Etienne Balibar. philosophie politique moderne. Karl Marx.

\section{Resumo}

O objetivo deste artigo é o de estudar a maneira na qual Louis Althusser elabora e aplica seu dispositivo de leitura aos clássicos do pensamento político moderno (Marx, Rousseau, Montesquieu) Com base nas críticas formuladas por Jacques Rancière, o artigo apresenta uma hipótese de pesquisa acerca da maneira na qual Althusser desenvolveu seu estudo da história da filosofia política. Assim, trata-se de questionar a dupla pertença deste autor: por um lado, militante do Partido Comunista Francês, pelo outro professor de filosofia na Escola Normal Superior, a que Balibar definiu a sua "singularidade intelectual". Sob esta ótica o trabalho analisa as estratégias e as contradições que Althusser desenvolve nas suas análises sobre os clássicos do pensamento político moderno. As questões mais especificas para as quais procura uma respostas são: como o filosofo francês organiza seu estudo do pensamento político moderno como "'uma propedêutica necessária para a inteligência de Marx"? ; Quais são as questões '(im)postas pela conjuntura' revisionista que orientam sua análise dos clássicos; e como seu dispositivo de leitura deles depende dos pressupostos marxistas e leninistas, elaborados a Por Marx e as introduções e Ler o Capital. Sendo assim, o artigo vai evidenciar como esses pressupostos constituem também os obstáculos epistemológicos ou limites da leitura que Althusser oferece da filosofia política moderna, isto a partir de sua tese mais celebre acerca da existência de um corte epistemológico na obra do Marx.

Palavras-chave: Louis Althusser. Jacques Rancière. Étienne Balibar. Filosofia Política Moderna. Marx. 
Wer den Feind will verstehen, muss in Feindes Lande gehen...

(Lénine, Matérialisme et Empiriocriticisme).

\section{Althusser et le dispositif de lecture des classiques}

"Althusser, c'est la fulgurance de quelques textes et l'éclat d'un échec" (Rancière, 1993, pp. 758-760). Par le biais de ce jugement lapidaire, Jacques Rancière lance en 1991 un avertissement aux interprètes, présents et futurs, afin de tracer une limite aux considérations posthumes à l'égard du marxiste, le plus célèbre, n'ayant jamais habité la rue d'Ulm. Lors de la première rencontre pour discuter l'œuvre d'Althusser après sa mort, Rancière veut se démarquer de l'approche qu'il voit prendre forme devant lui. C'est une approche qui, à ses yeux, aurait marqué l'althussérisme de façon dramatiquement évidente au moins à partir de 68. Plus concrètement, Rancière critique ceux qui, en insistant « sur le caractère ouvert et inachevé » de l'œuvre d'Althusser, mettent en avant l'évolution de sa pensée et les transformations de ses concepts» et nous invitent, ainsi, «à prolonger ce mouvement, à reprendre, tout en les rectifiant, les notions qu'il nous a laissées, à les refondre s'il est nécessaire et à les essayer dans des domaines nouveaux » (1993, p. 752-756). Nous pouvons aisément reconnaître aujourd'hui la prédominance de cette perspective herméneutique au sein des études althussériennes se développant au long des trente dernières années. Or, dès le début, Rancière considère que cette invitation vise a se "situer dans la communauté d'un work in progress», en revanche, trop indulgente avec Althusser. L'adoption de cette approche empêcherait de questionner les limites et les contradictions internes à l'œuvre dans les dispositifs de lecture que l'auteur de Pour Marx a forgés, et, ainsi, cerner les présupposés et les conséquences (aussi dans la Théorie !) des positions politiques qu’il a assumées.

Sur la base d'une critique 'gauchiste' de l'althussérisme - qu'il élabore en 1974 (Rancière, 2011) avec toute la sévérité d'un véritable 'j'accuse' contre la pédanterie de la Réponse à John Lewis - en 1991, Rancière vise, pourtant, moins à prolonger l'œuvre qu'à « interroger l'absence d'œuvre, ou le rapport tendu et violent 
entre l'œuvre et l'absence de l'œuvre » (1993, 764-765). Ainsi faisant, il s'attaque à un passage fondamental de l'Introduction à Lire le Capital avec l'objectif déclaré de déconstruire de l'intérieur le protocole de lecture que Althusser avait théorisé en l'occurrence sous le célèbre nom de 'lecture symptomale'1. En 1965 Althusser présente la lecture pratiquée au sein du séminaire comme 'coupable' parce qu'elle est conduite par des philosophes en quête de ce que Marx «n'aurait pas eu le temps d'exposer» en tant que tel, mais qui aurait cependant orienté et marqué de façon décisive l'entreprise, à savoir 'sa' philosophie (1997, p. 31). En l'occurrence, Althusser prévient ses lecteurs que l'interprétation proposée implique l'institution d'un cercle avec la conscience de ne pouvoir opérer qu'à son intérieur. Avec ses propres mots : «une lecture philosophique du Capital n'est possible que comme l'application de ce qui est l'objet même de notre recherche, la philosophie de Marx » (1997, p. 31).

Althusser définit l'institution de ce cercle 'indispensable' pour l'intelligence du philosophe de Trévire : «le cercle dialectique de la question posée à un objet sur sa nature, à partir d'une problématique théorique qui, mettant son objet à l'épreuve, se met à l'épreuve par son objet » (1997, p. 31). La recherche d'Althusser sur Marx vise ainsi l'articulation de la question épistémologique du matérialisme dialectique, en tant que science de l'histoire, à celle d'une philosophie «capable de rendre compte de formations théoriques et de leur histoire »(Althusser, 2005, p. 31). Afin d'accomplir sa mission, cette lecture 'coupablement' philosophique étudie la 'différence spécifique' de l'objet du Capital vis-à-vis de celui de la soi-disant 'économie classique' d'un côté, et de celui de la philosophie (idéologique) des Manuscrits de 1844 de l'autre. Cette double 'différence spécifique' développe la thèse de la 'coupure épistémologique', l'hypothèse herméneutique étant à la base des écrits recueillis dans le Pour Marx. Dans cet ouvrage Althusser s'approprie l'expression forgée par Gaston Bachelard², son but étant de décrire la construction par Marx de la critique de l'économie politique dans la perspective d'une césure avec ses propres

\footnotetext{
${ }^{1}$ Sur ce point : VINCENT, 1991, p. 1141-1146.

${ }^{2}$ Sur cette appropriation Balibar, 1991.
} 
écrits antérieurs (ceux dudit 'Jeune Marx'). Par 'coupure épistémologique' Althusser définit chez Marx le changement de la problématique théorique à partir de la fondation d'une nouvelle science (2005, p. 24). Dans cette perspective la singularité problématique de Marx reviendrait au fait que, chez lui, la fondation de l'histoire comme science tendrait à se confondre avec la révolution théorique pratiquée dans la philosophie (Althusser, 2005, p. 24). Ainsi, l'ambition d'Althusser devient celle de 'sauver' la philosophie marxiste d'une telle confusion et (surtout) de ses effets sur le marxisme ${ }^{3}$.

Plus généralement, la lecture collective du Capital réalisée en 1964-1965 vise la formalisation de la philosophie matérialiste, une tâche que Marx aurait laissée à sa postérité par 'manque de temps', c'est-à-dire par le fait de ne s'être consacré qu'à la découverte d'un nouveau 'Continent scientifique'. Ce nouveau continent est celui de l'histoire comme science des 'formations sociales', l'analyse du développement du mode de production capitaliste à partir de la lutte de classe (Althusser, 2005, p. 263264). Avec l'œuvre mature de Marx, ce nouveau continent se serait ajouté à ceux déjà connus de la géométrie et de la physique. En se constituant en paradigmes épistémologiques ces deux sciences avaient impulsé, dans le premier cas, la naissance de la philosophie (avec Platon) et dans le second, son profond renouveau avec la philosophie moderne (Descartes et Hobbes). La question qui anime Althusser devient alors : quels sont les effets de la découverte marxienne de l'histoire comme science pour la philosophie ?4 Le présupposé problématique qui soutient la lecture

\footnotetext{
${ }^{3}$ Il est notoire que déjà à partir des préfaces qu'il rédige pour les lecteurs italiens et anglais de Lire le Capital et puis dans une forme plus articulée avec la conférence Lénine et la philosophie, bien qu'il garde la thèse de la coupure épistémologique, Althusser procède à une autocritique qui débouche à une reformulation de la relation entre sciences et philosophie. Depuis la philosophie n'est plus saisie comme 'théorie de la pratique théorique' sinon comme 'lutte de classe dans la théorie'. Si, d'un côté la philosophie est toujours en retard, de l'autre - dans le cas de Marx - elle joue un rôle essentiel dans la production scientifique : "la révolution philosophique de Marx a commandé la 'coupure épistémologique' de Marx comme une de ses conditions de possibilité" (Althusser, 1976, p. 56).

${ }^{4}$ Et aussi : comment se transforment les articulations de la philosophie de l'histoire et I'histoire de la philosophie ? c'est une question qui occupe Althusser tout au long de sa vie comme en témoigne la sortie récente du volume posthume Ecrits sur l'histoire (2018).
} 
althussérienne de Marx est celui selon lequel le Capital constitue une 'révolution théorique' analogue à la révolution scientifique impulsée par la physique moderne de Galileo Galilei. En tant que telle la découverte scientifique de Marx imposerait après coup une mise à jour critique des catégories philosophiques. Il s'agit d'une révision des concepts opératoires que Rancière dénonce, par ailleurs, comme " police spéculative du philosophe fonctionnaire » (2011, p. 126) ou «politique de la science » (2011, p. 87). Pour Althusser cette sorte de aggiornamento de catégories et concepts ne peut se réaliser que par la théorisation de ce que Marx a produit au niveau de sa 'pratique'. De cette conviction découle son ambition de penser la philosophie comme 'théorie de la pratique théorique' présentée dans le Pour Marx.

Sur ce plan il devient manifeste que la perspective herméneutique althussérienne s'inspire d'une considération de Lénine, selon laquelle «la philosophie de Marx était toute entière contenue dans 'la Logique du Capital, cette Logique que Marx 'n'eut le temps' d'écrire» (Althusser, 1997, p. 27). C'est à partir de cette prémisse léninienne qu'Althusser définit comme objectif de leur séminaire l'analyse de la 'pratique théorique' de Marx. Il s'agit de la pratique aboutissant à la construction et à l'exposé (Darstellung) du Capital. Cela est loin d'être un cas isolé. Bien au contraire, les positions d'Althusser sur Marx trouvent presque toujours (au moins discursivement) leur source et leur orientation politique chez Lénine (Rancière, 2011, p. 72). Les interventions de ce dernier deviennent - au moins au niveau de la stratégie discursive althussérienne - l'exemple à suivre dans la définition d'une action politique dans la théorie. La philosophie se présente alors comme étant l'art de tracer des lignes de démarcation entre scientifique et idéologique. Ces lignes seraient nécessaires au prolétariat pour combattre sa lutte de classe dans la théorie ou, plus concrètement, pour définir ses stratégies politiques afin d’instaurer le communisme. A cet égard l'herméneutique althussérienne se présente au fond comme une formalisation sui generis de gestes théoriques léniniens ${ }^{5}$.

Or, dans 'la lutte de classe dans la théorie' de son temps, Althusser identifie l'adversaire principal dans la philosophie ayant sa source dans 'l'idéologie juridique'

${ }^{5}$ À ce sujet cf. MONTAG, 2013. 
bourgeoise, qui caractériserait la naissance des sciences humaines et la définition de leurs objets et méthodes. Tout au long du 20ème siècle, l’idéologie trouvant son paradigme dans le droit bourgeois tendrait à envahir et à s'approprier le champ marxiste. Les diatribes doctrinaires à l'origine de la naissance de la Troisième International, à savoir les lignes que Lénine trace pour se démarquer des positions de Kautsky, deviennent alors, pour Althusser, l'exemple à suivre pour ses propres interventions vouées à rectifier la ligne du Pcf d'une part, et (après 68) les dérives gauchistes de l'autre. Une telle offensive de l'idéologie bourgeoise dans le champ marxiste constitue le trait essentiel du 'réformisme' (révisionnisme de matrice libérale) contre lequel Althusser prétend combattre au sein du Parti (1976, p. 49). Sous cet angle son ambition devient celle d'élaborer les catégories de la philosophie marxiste pour affronter les nouveaux défis théoriques à l'époque de la déstalinisation. Althusser cerne, en effet, dans le $20^{\text {eme }}$ Congrès du PCUS la fin de l'époque du 'dogmatisme' marxiste (2005, p. 20).

Mais, comme souligne Rancière, le 'protocole' théorisé en 1965 n'est pas voué à être appliqué seulement aux textes marxiens. Ce protocole constituerait plutôt la formalisation d'un dispositif pour lire les classiques de la pensée politique moderne à partir des 'abstractions différentielles', autrement dit les 'décalages' dans la théorie que les auteurs auraient produit vis-à-vis de la pensée dominante de leur temps. Comme le suggère Rancière, le cas exemplaire de ce protocole d'analyse des classiques modernes est l'écrit publié dans les Cahiers pour l'analyse sous le titre Sur le Contrat social en 1967 (1993, p. 1071). Retravaillant pour publication une partie de son cours du semestre 1965-66, Althusser analyse dans cet essai les 'décalages’ que Rousseau aurait produit vis-à-vis des doctrines du droit naturel. Cela à partir de la façon dont Rousseau s'approprierait la figure centrale de cette tradition de pensée, à savoir le 'contrat' (Althusser, 1998, p. 61). La lecture qu'Althusser engage des classiques de la philosophie politique moderne part de l'idée que leur œuvre constitue la 'préhistoire' de la révolution théorique mentionnée. L'étude de la pensée du 18 ème siècle est nécessaire parce qu'elle est 'propédeutique' pour 'l'intelligence de Marx'. En cernant la philosophie classique comme préhistoire, Althusser s'inspire d'une phrase 
que Marx écrit dans l'Introduction de 1857: «L'anatomie de l'homme est la clef de l'anatomie du singe » (1980, p. 40). Cette phrase nous donne la perspective d'une histoire de la philosophie à développer à partir de la présupposition que Marx ait effectivement réalisé une révolution théorique. Autrement dit, l'anatomie de la philosophie de Marx devient la clef pour saisir l'anatomie de la philosophie moderne.

Or, bien que la littérature critique ait généralement ignoré la manière dont Althusser approche les classiques de la pensée politique, à nos yeux, cette question est tout à fait centrale pour sonder la limite interne de son dispositif d'énonciation. Il nous revient ainsi d'étudier son point de vue dans l'analyse des philosophes du $18^{\text {ème }}$ siècle. C'est justement afin de cerner cette limite que nous nous appuyons sur la critique de Rancière. Nous faisons cela sans pour autant y souscrire dans sa totalité, ni partager son positionnement politique. Vouée à relativiser la 'fulguration' de certaines thèses pour mettre en lumière 'l'échec' d'ensemble de cet engagement qui se prétendait collectif, l'examen 'savant' auquel Rancière soumet en 1991 l'Introduction de Lire le Capital part d'un passage décisif pour comprendre la ‘fascination' exercée par Althusser sur la génération étudiante française d'avant 68 : «Les brefs éclairs des Thèses sur Feuerbach frappent de lumière tous les philosophes qui les approchent mais chacun sait qu'un éclair éblouit plus qu'il n'illumine et que rien n'est plus difficile à situer dans la nuit qu'un éclat de lumière qui la rompt » $\left(2005\right.$, p. 28) ${ }^{6}$.

Cette phrase ayant aussi suscité en lui plein d'espoirs à l'époque de sa publication, devient, en 1991, l'épitaphe que Rancière veut entailler sur la tombe de son auteur (1993, pp. 1123-1226). Pour Rancière, « il faut en prendre acte : pour le meilleur et pour le pire, Althusser a d'abord été un enchanteur, un éclair éblouissant et aveuglant qui est retombé dans la nuit » (1993, pp. 765-766). En l'occurrence, afin d'exorciser l'hypocrisie caractérisant souvent les commorations, Rancière ne fait pas taire le doute que l'œuvre d'Althusser ait aussi un lien avec la maladie marquant sa vie ainsi qu'avec l'accueil de ses écrits après 1980. Sous les effets de ce malaise

\footnotetext{
${ }^{6}$ Cette idée est reprise et développée dans l'Introduction de Lire le Capital (Althusser, 1997, p. 26).
} 
psychique, notamment, le 16 novembre 1980, Althusser étrangle sa femme. Par ce biais, il se condamne à la sortie de la scène publique, à l'ignominie et à la malédiction. Par la sentence de 'non-lieu' le tribunal déclare le philosophe auteur d'actes non imputables à sa volonté l'obligeant ainsi au silence pendant la dernière décennie de son existence ${ }^{7}$. Lorsqu'il prend la parole en 1991, Rancière convient avec les autres participants du colloque de l'opportunité d'émanciper la pensée d'Althusser d'une telle triste (et au fond injuste) condamnation à l'oubli. Néanmoins, afin de réhabiliter publiquement la parole de son ancien maître, Rancière insiste sur la nécessité de poser une condition, celle de prendre la mesure des limites dans l'énonciation de ses thèses.

\section{Althusser et la pratique de l'histoire de la philosophie}

Combien la dépression ou la prédisposition au délire d'Althusser ait pu influencer sa production scientifique est un problème de longue date, autant incontournable qu'insoluble. Sous cet aspect nous n'avons qu'à accepter l'invitation formulée par Althusser dans L'Avenir dure longtemps. Selon lui, il ne faut pas chercher dans une analyse de ce genre le dernier mot sur le sens objectif d'une philosophie. A son dire, en effet, « quelles que soient les motivations internes, conscientes ou plutôt inconscientes de tout philosophe, sa philosophie écrite est une réalité objective, y passe tout entière, et ses effets ou non sur le monde sont des effets objectifs qui à la limite, n’ont plus aucun rapport avec cet intérieur que je décris ». (1991, p. 167).

Ainsi, nous n'avons qu'à étudier une telle 'réalité objective', à savoir ce qui nous est resté d'Althusser au-delà de ses vicissitudes personnelles. Il s'agit des écrits qu'il a signés de son vivant aussi bien que des notes et manuscrits qui sont en train d'être publiés à partir de son leg déposé à l'IMEC. Dans l'analyse de ses documents il ne nous revient pas de juger de la pertinence des positions assumées par les protagonistes, ni l'opportunité de leurs prises de parole publiques. Par ailleurs ce

\footnotetext{
${ }^{7}$ Sur ce point les paroles de Balibar (1991, p. 59-60).
} 
type de questions semble avoir occupé plus l'esprit de ses anciens élèves que celui du vieux maître ${ }^{8}$. Notre réflexion nait plutôt de la conviction que l'épitaphe de Rancière ouvre une perspective éclairante afin d'analyser un aspect de l'activité d'Althusser peu étudié jusqu’à présent. Un aspect que, en revanche, nous considérons décisif non seulement pour comprendre Althusser et certains effets - en partie 'souterrains' (1994a) - de son œuvre, mais aussi pour questionner notre pratique d'enseignantchercheurs dans le domaine de l'histoire de la pensée politique.

Comme 'caïman' la tâche principale d'Althusser était celle de préparer les élèves de l'ENS au concours d'agrégation. Ses cours portaient surtout sur la philosophie politique de Machiavel à Rousseau. Ainsi, Althusser a marqué les esprits d'une génération d'excellent enseignants-chercheurs en philosophie (Balibar, Macherey, Moreau, Rancière, Tosel...). Certaines des 'fulgurations' althusseriennes ont inspirés des relectures et études originales des classiques de la philosophie politique moderne bien au-delà du cercle rapproché de ses élèves. Par ses cours et essais, Althusser a non seulement influencé ses élèves directes mais il continue aussi à stimuler ses lecteurs. Il est devenu une référence presqu'incontournable dans les études sur Machiavel et Rousseau.

Notre tâche devient alors celle de questionner les limites et la puissance du dispositif de lecture althussérien quant aux classiques de la philosophie classique à partir des présupposés théorisés en 1965. Ainsi, à partir de l'exemple livré par l'auteur de Pour Marx, nous croyons pouvoir interroger le rapport de l'historien de la pensée politique avec sa pratique. Pour cela il faut, d'un côté, analyser les protocoles de lecture althussériens à partir de la criticité de leurs présupposés 'communistes' ou 'marxistes' et, de l'autre, de thématiser le rapport au présent c'est-à-dire aux problèmes (im)posés 'par la conjoncture' avec la pratique d'enseignement des classiques dans le cadre institutionnel de la préparation aux concours publiques.

A présent, le lecteur - même celui peu avisé avec Althusser - pourra être perplexe vis-à-vis de notre démarche. Suivant une image assez répandue, Althusser

\footnotetext{
${ }^{8}$ Althusser reconnait à Rancière un rôle décisif dans la réussite du séminaire de 1964-65 (1991, p. 200-201).
} 
n'aurait pas été ni voulu être un historien de la pensée politique. Ou bien sa pratique d'enseignement ne répondrait qu'à une obligation institutionnelle et, pourtant, elle ne devrait pas être évaluée à partir de sa théorisation de la pratique théorique. Tout cela a ses raisons d'être pensé. Toutefois, notre lecteur pourra bien nous concéder de pouvoir considérer les contradictions (entre théorie et pratique) mentionnées comme l'expression (éventuellement la plus superficielle) de quelque chose que Balibar a néanmoins défini comme la «singularité intellectuelle Althusser». Celle d'avoir été « en même temps totalement philosophe et totalement communiste sans sacrifier, sans subordonner, sans assujettir aucun des termes à l'autre » (1991, p. 121).

A bien voir la tentative de tenir une telle position intellectuelle conditionne et façonne toute l'œuvre d'Althusser'. Sa centralité devient manifeste dans le célèbre incipit de la Soutenance d'Amiens où l'auteur prétend présenter le fil conducteur de son activité et de sa production scientifique. En l'occurrence il mentionne les projets d'une « grande » et d'une " petite Thèse » rendues dans les mains de Jean Hyppolite et Vladimir Jankélévitch entre 1949 et 1950. La grande thèse aurait dû porter sur Politique et philosophie an $18^{\text {ème }}$ siècle tandis que la petite thèse aurait eu à traiter du Deuxième Discours de Rousseau. En présentant ses travaux devant le jury, Althusser déclare : «J'étais déjà communiste, et l'étant, je tentais aussi d'être marxiste ; c'est-àdire j'essayais, comme je pouvais, de comprendre ce que le marxisme veut dire. Ainsi ce travail sur philosophie et politique au $18^{\text {ème }}$ siècle, je l'entendais comme une propédeutique nécessaire à l'intelligence de Marx » (1998, p. 201).

Notre questionnement porte sur cette double appartenance - militant du Parti communiste français d'un côté, et professeur de philosophie à l'ENS de l'autre - avec le but d'investiguer les stratégies, les synergies et les contradictions qu'Althusser développe dans ses recherches et pratiques d'écriture sur les classiques de la pensée politique moderne. Cela dans la tentative de concilier ou articuler ces

\footnotetext{
${ }^{9}$ "J'essayai de concilier cette critique radicale de la philosophie comme imposture idéologique [...] avec mon expérience de la pratique philosophique, et aboutis d'abord à des formules du genre : 'la philosophie représente la science auprès de la politique et la politique auprès de la science', et plus tard : 'la philosophie est, en dernière instance, lutte de classes dans la théorie' » (Althusser, 1991, p. 161).
} 
deux versants de son existence quotidienne. Ainsi, les questions auxquelles nous tâchons de répondre sont: comment organise-t-il son étude de la philosophie moderne comme une « propédeutique nécessaire à l’intelligence de Marx ? »; Quels sont les problèmes, 'imposés' par la conjoncture 'révisionniste', qui influencent son analyse des auteurs classiques? Comment son enseignement de l'histoire de la philosophie politique est-il orienté par le présupposé suivant lequel Marx aurait réalisé une révolution théorique avec le Capital?

Bien qu'exprimant des jugements très différents entre eux, Rancière et Balibar conviennent néanmoins sur un point: pour le meilleur et pour le pire, Althusser a pleinement assumé son rôle institutionnel de maître ${ }^{10}$. Il s'agit d'un rôle voué aujourd'hui à disparaitre car insondable par les dispositifs qui ont été choisis pour l'évaluation des professeurs. Althusser s'était intéressé moins aux lignes à ajouter à son cv, qu'à l'institution d'une communauté de recherche. C'est précisément dans cette optique que son exemple intempestif peut nous servir de contre- modèle pour questionner notre pratique d'historiens de la philosophie politique.

Or, selon Rancière, le cœur de l'althussérisme est 'l'éducation des éducateurs' et ce dans le double registre d'éducation universitaire d'un côté et éducation militante de l'autre. Une éducation pensée comme moment propédeutique à toute transformation véritablement révolutionnaire dans la théorie ainsi que dans la pratique (2011, p. 81). À ses yeux la posture magistrale d'Althusser devient autant évidente qu’insupportable dans la célèbre Réponse à John Lewis. Althusser incarnerait ce 'sujet supposé savoir' pilier de la fonction pédagogique autoritaire au crible des critiques exprimées par le mouvement soixante-huitard (Rancière, 2011, p. 192).

Cependant, nous disposons aussi d'éléments suffisants pour affirmer qu'Althusser incarnait également certains traits du 'maître ignorant' théorisé par

\footnotetext{
${ }^{10}$ "Dans l'Université, dans les Lettres, dans la Politique, je vois des solitudes et des mimétismes, des pédagogues généraux et des exploiteurs sans scrupules, des inspirateurs et des organisateurs, mais je ne vois pas d'intellectuels pour qui, comme pour lui, le partage ait été la condition même de la pensée. Peut-être n'eut-il aucun mérite à cela, car je crois que c'était chez lui un besoin. Althusser avait dû naître partageux. C'est pourquoi, moi qui comme toute une génération ait tout appris, sinon de lui, du moins grâce à lui, je ne trouve pas que le nom de 'Maître' lui convienne bien »(BALIBAR, 1991, p. 120-121)
} 
Rancière sur l'exemple de Jacotot ${ }^{11}$. Parmi ces traits les plus importants et indiscutables est le mérite d'avoir initié, ou, au moins, rendu possible le mouvement d'appropriation collective de l'œuvre de Marx par l'organisation du séminaire de lecture du Capital à l'ENS (Rancière, 1993, p. 766-772). Cette expérience se réalisant en absence d'un guide magistral marque profondément toute une génération même au-delà des cercles de l'ENS. Suivant les récits des participants le séminaire non seulement naquit sur demande de certains élèves mais il aurait aussi fonctionné sur la base du 'principe d'égalité des intelligences' tant cher à Rancière ${ }^{12}$.

A vrai dire la critique de ce dernier se focalise sur une autre attitude indubitablement présente chez Althusser. Après la lecture de son autobiographie, il devient difficile de nier (ou négliger) que l'affirmation selon laquelle ce sont les masses et non pas les hommes qui font l'histoire, n'empêchait pas Althusser de se réfugier dans la 'solitude' que lui assuraient les murs de l'ENS. Ou que, au moment de se passionner pour l'héroïsme théorique des auteurs du passé (Rancière, 2011, p. 73), il n'aurait pas nourri l'ambition de les émuler (Althusser, 1991, p. 165). Pour Althusser la philosophie matérialiste se produit 'dans le vide d'une distance à prendre' (correspondant aux 'abstractions différentielles') et son histoire consiste dans les démarcations tracées par les grands philosophes de l'histoire (ceux que Rousseau nommait « vrais savants ») (Rousseau, 1964, p. 39).

C'est à ce geste au fond solitaire, à savoir dans la production de décalages autant décisifs que littéralement inconsistants vis-à-vis de la pensée dominante de son temps, que tient la possibilité de tracer de telles lignes de démarcations vouées à ouvrir le chemin pour une action émancipatrice. Cette action coïncide avec le communisme entendu comme abolition (dynamique) de l'état de choses présent.

\footnotetext{
${ }^{11}$ Cf. RANCIERE, 1987, p. 68.

12 Selon Balibar: "Avec le temps, avec ce qu'on appelle l'expérience, j'émerveille de l'aptitude unique qu'Althusser eut de ne pas travailler seul, d'inclure les autres dans son travail, sans les contraindre ni se contraindre lui-même (la contrainte venait d'ailleurs des choses elles-mêmes), allant jusqu'à différer d'écrire, lui qui n'a jamais écrit que dans l'urgence, allant jusqu'à transformer son projet pour attendre d'être rejoint par des collaborateurs, pour faire avec eux une œuvre intellectuelle commune » (1991, p. 120). Pour Rancière il s'agit, cependant, d'une intelligence commune reconnue par le concours d'admission à l'École.
} 
Néanmoins, dans sa tentative de rester fidèle à la lettre et à l'esprit d'Engels, Marx et Lénine, celui qui déclarait être attiré par les forêts des classiques, nous aurait-il livré finalement que des récits troublants et déserts doctrinaires?

Après ces considérations le lecteur le plus indulgent pourra peut-être encore nous concéder d'être en train de toucher un point crucial de la pensée et de l'héritage intellectuel d'Althusser. Toutefois, une fois arrivés à ce point, il ne pourra pas s'empêcher non plus de nous adresser une autre objection vouée inéluctablement à invalider l'articulation de notre propos: Althusser n'a jamais thématisé 'l'histoire de la philosophie' comme objet théorique ! Au contraire, dans le sillage des indications contenues dans l'Idéologie allemande, une de ses thèses les plus chères était celle selon laquelle la philosophie (en tant que métaphysique) n'a pas d'histoire! L'historicisme est avec l'humanisme et l'économicisme à la base du révisionnisme bourgeois que Althusser avait identifié comme adversaire dans la lutte de classe dans la théorie (1976, p. 47). Dans l'œuvre de Marx il lui revient d'extraire', de 'dégager' sa philosophie car, comme le disait Lénine, elle est 'vraie', et cela ne se prête à aucune discussion.

Sans doute ce 'parti pris' définit souvent le point de vue d'Althusser sur les classiques. Ce choix préalable et littéralement dogmatique se traduit dans la recherche, chez ces auteurs, de questions ou signes que Marx aurait enregistrés pour les réélaborer en vue de sa révolution théorique. Cependant, en même temps, les présences de la philosophie moderne dans l'œuvre de Marx représenteraient aussi des brèches par lesquelles l'idéologie bourgeoise peut s'approprier à son tour le marxisme tout en neutralisant son potentiel révolutionnaire. Par son invasion du camp marxiste l'action de l'idéologie bourgeoise désarticule l'union entre théorie et pratique révolutionnaires que Lénine indiquait comme conditio sine qua non pour toute action communiste véritablement efficace.

Or, 'l'Humanisme théorique' ainsi que l'Historicisme' ou 'l'Économicisme' sont des personnages textuels, c'est-à-dire des adversaires construits ad hoc par Althusser afin de déconstruire la prétention de scientificité propre à l'idéologie dominante. Celle-ci, s’imposant comme vérité universelle, façonne l'obédience aux 
rapports de force existants. Par ce façonnement réalisé par les Appareils Idéologiques d'Etat se renforce et se perfectionne la dictature de la bourgeoisie (dont la dictature du prolétariat détiendrait le secret) (Althusser, 2016, p. 2039). D’un côté, cette position formalise la critique que Marx adresse à la Déclaration des droits de l'bomme et du citoyen. En instituant le 'Tribunal de la raison' le paradigme de la philosophie moderne et de l'idéologie juridique semble pourtant trouver son expression, la plus rigoureuse, dans l'œuvre de Kant (Derrida, 1990, p. 72-104). De l'autre, Althusser ambitionne de formaliser la différentiation de Marx vis-à-vis de l'économie politique (classique) en historicisant les catégories que les 'économistes classiques' prétendaient intemporelles (1997, p. 272).

Or, le parti pris mentionné parait, en réalité, opérer comme limite intérieure au discours d'Althusser, se situant à l'origine d'incessants anachronismes et aveuglements qui prennent la forme d'une distorsion historico-conceptuelle quant aux problèmes à traiter. En ce sens un passage emblématique est contenu dans le livre sur Montesquieu, là où Althusser soutient (contre les anachronismes de la littérature libérale) :

\begin{abstract}
II ne faut pas se dissimuler que les contemporains vivaient leur histoire en la pensant, et que leur pensée encore à la recherche de critères scientifiques manquait du recul nécessaire qui permet à la pensée de devenir critique de la vie. Pensant une histoire dont les ressorts profonds leur échappaient, ils s'exposaient à borner leur pensée aux catégories immédiates de leur vie historique, prenant le plus souvent des intentions politiques pour la réalité même, et des conflits de surface pour le fond des choses (1959, p. 112).
\end{abstract}

Dit autrement, d'un côté Althusser cherche dans les classiques de la pensée moderne les signes précurseurs et les outils conceptuels de la révolution théorique que seulement Marx réaliserait avec le Capital. Et de l'autre il cherche aussi les positions à partir desquelles il faudrait mesurer les décalages dans la philosophie que cet ouvrage aurait produit ou serait en train de produire dans la durée. Mais, par le simple fait que Marx ne nous a laissé aucun exposé de sa philosophie, aux yeux d'Althusser, l'histoire de la philosophie prend les allures d'un champ de bataille (Kampfplatz) (1998, p. 201) dans lequel ne s'affrontent que deux courants, à savoir l'idéalisme et le matérialisme. Or, dans ce contexte, la révolution théorique de Marx n'aurait pas encore produit d'effets décisifs. En bref, la révolution scientifique 
marxienne n'aurait produit aucun bouleversement paradigmatique sur la pratique de la philosophie.

Par conséquent matérialisme et idéalisme s'affrontent encore dans le cadre d'une substantielle continuité sous l'hégémonie de la philosophie du sujet et de ses garanties. Althusser considère 'le sujet de droit' comme la catégorie basique de la pensée moderne. La coupure reste à faire, constituant une tâche qui s’identifie avec la pratique philosophique du matérialisme. À la philosophie matérialiste revient, portant, la tâche de s'opposer, ou mieux, de se démarquer de façon continuelle, par la production de décalages ponctuels vis-à-vis des positions de la pensée ou idéologie dominante. Cette production de décalages ponctuels est orientée par des 'Thèses' qui constituent des mots d'ordre dans la pratique théorique tout en garantissant sa 'fidélité à la ligne'. Sous beaucoup d'aspects Althusser trouve dans les classiques du marxisme des formules qui le guident dans l'esquisse de ses thématiques, choisies ou imposées par la conjoncture, avec l'objectif d'en renouveler les contenus (paradoxalement, pour quelqu'un qui se disait matérialiste, d'en renouveler la lettre sans en trahir l'esprit).

Pierre-François Moreau, l'ancien élève qui s'est occupé le plus de la philosophie à l'âge classique, a souligné que :

La philosophie pour Althusser est une pratique de la division; ce qui explique qu'il soit impossible d'en faire l'histoire puisqu'elle ne se définit pas à partir des contenus qui s'opposent. Au contraire elle se situe dans la frontière entre deux camps non philosophiques qu'elle sépare en traçant entre eux une ligne de démarcation (1997, p. 78).

Par-là l'histoire de la philosophie devient un simple théâtre dans lequel les philosophes se retrouvent l'un à côté de l'autre, chacun se caractérisant par une thèse, un concept, une catégorie ou un problème. Un exemple emblématique de cette conception est la scène surréaliste par laquelle Althusser ouvre son manuel Être marxistes en philosophie. Socrates, Hobbes, Hegel, Hume, Platon, Spinoza sont tous réunis dans une discussion dans laquelle chacun insiste sur l'objet théorique caractérisant sa propre pensée. Et tous sont mis en échec par Lénine (Althusser, 2015, p. 55-71). Cette mise en échec vient pourtant de l'extérieur, du 'dehors' de la 
philosophie: la politique. L'objectif devient ainsi d'élaborer une 'théorie nonphilosophique de la philosophie'. Il s'agit d'une idée qu'Althusser expose avec plus de détails dans la conférence Lénine et la philosophie (1998, p. 107).

Mais le théâtre mentionné montre bien également que, suivant Althusser, chaque philosophe peut être étudié à partir de son objet spécifique. Ainsi, sa singularité reviendrait à sa façon de construit son objet théorique ou sa problématique (Balibar, 1993, p. 1397). Une fois que l'on cerne la façon dont un (vrai) philosophe construit son objet, à savoir la façon dont il s'approprie une question livrée par la tradition ou imposée par la conjoncture, cette appropriation singulière devient, pour son lecteur, la sonde (la 'carotte') permettant de pénétrer dans sa pensée (Althusser, 1991, p. 159).

\section{Marx malgré Marx}

Aussi bien dans les écrits publiques et privés que dans les cours d'Althusser, d'autres auteurs y jouent un rôle important que ceux auxquels il consacre ses essais (Machiavel, Marx, Montesquieu ou Rousseau). Nous pensons à Hobbes, Helvétius et, surtout, à Spinoza. C'est a ce dernier qu'Althusser s'identifie dans son travail philosophique. À ce propos Moreau suggère que l'on peut identifier en Spinoza le véritable précurseur de la position défendue par Althusser vis-à-vis de l'histoire de la philosophie. Cela parce que chez Spinoza aussi, au long des années, la philosophie indiquerait plus le lieu de la polémique qu'un contenu réel. Pour lui aussi la philosophie se produirait par des démarcations vis-à-vis de la non-philosophie, le sens commun ou la théologie (secret de l'idéologie religieuse chez Althusser). En outre, Moreau rappelle que pour Althusser l'inconsistance de l'historiographie de la philosophie française après Victor Cousin était le signe de l'incapacité de la philosophie bourgeoise d'écrire sa propre histoire. Néanmoins il est difficile de soutenir que cet aspect ne caractérise pas au fond aussi la philosophie matérialiste d'Althusser dans sa pratique de division et opposition à l'idéologie bourgeoise : 
Cette division se fait donc sur place et non à l'intérieur d'une progression historique, et I'histoire de la philosophie est seulement la répétition de cette même division, non l'histoire d'un contenu. Cette division prend la forme d'une césure entre matérialisme et idéalisme, et même si le matérialisme se modifie avec l'histoire des sciences la division est au fond toujours la même, ce qui fait que «pour l'essentiel la philosophie n'a pas vraiment d'histoire » (Moreau, 1997, p. 79).

Or, suivant Moreau, cette position explicitement antihistoriciste permettrait de manière paradoxale à Althusser de produire un décalage voué à renouveler la discipline de l'histoire de la philosophie de son intérieur. Ce décalage ouvrirait une histoire de la pensée comme 'histoire d'événements' (de révolutions théoriques ou politiques). Si nous intégrons cette suggestion de Moreau à notre hypothèse de recherche nous pouvons conjecturer qu'Althusser esquisse, sans doute inconsciemment, une histoire de la philosophie composée de grands événements et de micro-événements, gestes singuliers et imperceptibles accomplis par de 'vrais philosophes' vis-à-vis de la pensée dominante. Suivant la ligne à peine esquissée qui accompagne la surdétermination de la sous-détermination ${ }^{13}$, ces événements garderaient la virtualité d'autres possibles. Les décalages théoriques garderaient la capacité d'impulser des déviations potentiellement décisives s'ils contribuent à renouveler la théorie en se branchant (aléatoirement) à des pratiques révolutionnaires singulières. Voilà comment apparaît l'horizon du matérialisme aléatoire, un sujet sur auquel Althusser dédie ses réflexions dans l'isolement pendant ses 'années d'hiver'.

Or, l'analyse de ces tendances souterraines en tant que 'sous-déterminées' profilerait un renouveau de l'étude de l'histoire de la pensée politique en vue d'une praxis finalisée à la transformation du statu quo. La relation entre politique et pratique de l'histoire de la philosophie ainsi esquissée aurait aussi un impact sur l'organisation des cours. Et cela deviendrait évident au niveau de leurs variations. C'est ce que Moreau semble suggérer avec perspicacité en analysant le Machiavel et nous. En 1972 l'intérêt qu'Althusser nourrit pour la question de l'État serait à l'origine de la place prise par Machiavel dans son cours (Moreau, 2001, p. 148).

\footnotetext{
${ }^{13}$ Au sujet de la sous-détermination voire Balibar dans son Avant-Propos à l'édition de 1996 de Pour Marx (Althusser, 2005, pp. XII-XIII).
} 
Dans l'optique althussérienne le progrès caractérise l'histoire des sciences. De cette dernière dépendent les modèles scientifiques que la philosophie adopte. Or, la science n'appartient pas aux anciens mais elle n'est que moderne. De cette façon, la philosophie élabore les concepts que la science rend disponibles et qu'elle diffuse par ses découvertes et révolutions. Ces dernières soutiennent les formations théoriques dominantes (science, idéologie, philosophie) dont Althusser formalise l'histoire à partir de l'analyse des pratiques théoriques de Marx et Lénine. L'histoire de la philosophie devient l'histoire du théorique (et elle est ainsi 'sauvée'14 de la critique à la métaphysique contenue dans l'Idéologie allemande). Nous avons déjà mentionné comment la référence marxienne et le pari de sa révolution théorique ont pu provoquer chez Althusser certains effets d'aveuglement sur sa lecture des classiques, analogues à ceux venant de l'idéologie bourgeoise. En cherchant 'l'anatomie' de la philosophie du 18 ème siècle chez Marx, Althusser n'a finalement pas conduit ses analyses à partir d'un évident anachronisme prospectif. À cet égard l'étude de la manière dont Althusser approche les classiques de la philosophie politique moderne est sans doute essentiel pour cerner la limite interne de son protocole de lecture.

Dans son autobiographie Althusser écrit que plus il lisait et étudiait Marx, plus il s’apercevait «que Marx avait pensé, le sachant ou non, dans des pensées de grande importance dont les auteurs l'avaient précédé : Épicure, Spinoza, Hobbes, Machiavel (partiellement à vrai dire) Rousseau et Hegel» (1991, p. 203). De cette façon il se serait convaincu «de plus en plus que la philosophie de Hegel et Feuerbach avait servi à la fois de 'point d'appui' et d'obstacle épistémologique au développement de ses propres concepts jusque dans leur formulation » (1991, p. 203). La scène textuelle construite par Althusser devient alors celle d'un Marx qui lutte contre lui-même. De ce point de vue, l'ambition d'Althusser paraît d'avoir été celle de prospecter moins un Marx au-delà de Marx, qu'un Marx malgré Marx. Cette

\footnotetext{
${ }^{14}$ Selon Rancière, le véritable 'enjeu' d'Althusser et de l'althusserisme serait celui « de sauver la philosophie et la philosophie marxiste en particulier comme affaire des spécialistes universitaires » (2011, p. 41).
} 
difficulté dans la lecture de Marx découle (en dernière instance) de l'hypothèse fondamentale d'Althusser, celle de la coupure épistémologique. À ce sujet deux points doivent maintenant être soulignés : le premier, que la coupure décrit aussi une pratique de subjectivation; le second que sa datation a des conséquences importantes sur l'interprétation du rapport Marx-Hegel. Dès sa première formulation dans le Pour Marx la coupure implique, en effet, aussi un certain type de subjectivation personnelle. Avant tout, la subjectivation de Marx avec la critique de l'économie politique s'accompagne d'une prolétarisation matérielle et politique (Althusser, 1976, p. 56-57). Au centre de ce processus se produit la coupure avec l'Idéologie allemande dans laquelle Marx avait baigné pendant toute sa formation théorique. Mais la coupure dénoterait également un défi pour une la génération d'Althusser : celui de rompre avec la fascination pour le jeune Marx comme si dans les Manuscrits était contenue toute sa philosophie (Althusser, 2005, p. 17-18).

Cet aspect subjectif de la coupure va ainsi se nouer avec une des raisons les plus profondes de la passion d'Althusser pour Machiavel et son Prince qu'il interprète comme 'acte politique' dans la théorie (2009, p. 62). Dans le sillage de Gramsci, Althusser cerne cette œuvre comme un 'manifeste' dans lequel Machiavel «se-fait peuple» (2009, p. 64). Et justement avec le devenir-peuple (ou prolétaire) se joue en grosse partie le rapport critique avec Rancière et les maö̈stes français. Cette subjectivation personnelle par le biais de la pratique théorique semble décrire, pour Althusser, le geste que la philosophie matérialiste doit accomplir de façon incessante. Voici la modalité d'existence de la philosophie : couper, se démarquer, produire un décalage pertinent. A cet égard la coupure dénote moins une rupture d'époque que l'horizon de la pratique philosophique matérialiste à l'œuvre. C'est bien en ce sens qu'Althusser parle de 'coupure continuelle' (Althusser, 1976, p. 53).

Or, lorsqu'il date la coupure Althusser avise son lecteur que son choix aurait eu des conséquences décisives sur le rapport de Marx avec la philosophie du droit de Hegel (2005, p. 29). Lorsqu'il défend ce choix, Althusser présente les Manuscrits comme un simple développement de la critique de Feuerbach à la religion. Selon lui, Marx se limiterait à appliquer cette critique au droit et à la politique. La conséquence 
directe de la datation d'Althusser est cependant celle de réduire et affaiblir la critique de Hegel au droit naturel. Il ne fait aucun doute que la thèse de l'existence d'une coupure 'sans équivoques' (Althusser, 2005, p. 29) implique un 'forçage'. Par ailleurs, c'est exactement là que réside la force d'une thèse en philosophie : elle doit impulser un mouvement, dans ce cas spécifique celui d'une approche critique à l'œuvre marxienne dans le cadre d'une appropriation singulière. Mais cette posture a aussi comme conséquence la production de bévues et aveuglements chez Althusser luimême. Suivant Rancière, tout en voulant marquer une séparation, Althusser arrive à attribuer à Feuerbach des thèses du jeune Marx (2011, p. 34). Dans le théâtre de la coupure les personnages textuels tendent, ainsi, à dépasser les frontières des auteurs traités par Althusser. Loin d'éliminer les problèmes, ni la nécessité de leur articulation, ce point nous signale simplement les effets de confusion étant en jeu.

L'adoption d'une perspective historico-conceptuelle permet de cerner la limite dérivant de la coupure dans la saisie du rapport entre Marx, Hegel et la critique du jusnaturalisme. L'hypothèse euristique de l'histoire conceptuelle tient à l'existence, dans l'histoire de la pensée politique, d'une séparation (Trennung), voire d'une coupure. Suivant Koselleck, les concepts politiques modernes se formeraient

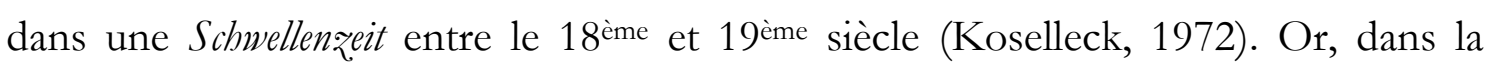
formalisation euristique de Koselleck y joue un rôle central la compréhension hegelienne du droit naturel moderne. Hegel récupère l'idéal de la Polis grecque pour penser le dépassement de la philosophie intellectualiste du 18 ème siècle (Duso, 2013). Or, animé comme il est par la volonté de souligner la rupture de Marx avec des prédécesseurs Althusser essaie à tout prix d'affaiblir la critique de Hegel à la pensée politique moderne. Il s'agit pour autant d'une critique que Marx intègre à sa part destruens. Le désaccord de Marx avec Hegel tient à la théorisation de l'État tandis que la critique du dispositif jusnaturaliste se laisse au fond cerner dans le cadre d'une continuité (critique au 'formalisme juridique'). Selon notre hypothèse la datation de la coupure peut aider à comprendre les difficultés rencontrées par Althusser lorsqu'il critique le formalisme juridique. Son dernier combat dans les années soixante-dix se focalise sur la critique de l'abandon de la dictature du prolétariat du programme du 
Pcf. Dans Marx dans ses limites, Althusser dénonce la crise des institutions bourgeoises et des modèles organisationnels du 20ème siècle (Parti, État, démocratie représentative) (1994b, p. 473-486). Dans la réédition d'Idéologie et Appareils Idéologiques d'Etat en 1976 il nomme ce système idéologique «Appareil Idéologique d’État politique ») (2011, p. 252-256).

Voici donc notre hypothèse. Un questionnement historico-conceptuel de la pratique althussérienne de l'histoire de la philosophie permet de cerner et mettre en lumière le cercle qui surgit avec son pari théorique de matrice leninienne. Ce pari est celui de croire que Marx ait réalisé une révolution théorique. Pour faire court : l'idée serait que pour penser la coupure chez Marx Althusser affaiblit les effets de la formation des concepts politiques modernes. Cette relativisation de leur portée contribuerait aux difficultés qu'il rencontre dans la formalisation de la philosophie matérialiste.

De ce point de vue la limite du marxisme d'Althusser semble coïncider une fois de plus avec son léninisme. L'exemple de Lénine oriente et inspire Althusser dans ses analyses et dans la formulation de ses thèses révolutionnaires. En même temps il parait aussi l'aveugler au moment de repérer chez Marx les virtualités qui n'ont pas de suite dans l'expérience bolchévique. Sa critique de la dictature du prolétariat suit pas à pas le chemin léninien (qui s'appuie sur la Critique du programme de Gotha) sans mentionner presque jamais les écrits de Marx sur la Commune (Althusser, 2016). Enfin, la fidélité à un certain Lénine semble constituer le 'nom propre' de la limite interne opérative dans la lecture de Marx par Althusser et, par conséquent, des classiques de la philosophie politique moderne, et bien sûr aussi dans sa tentative (par ailleurs louable et qui reste toujours à faire) de renouveler le marxisme.

\section{Bibliographie}

Althusser, L., 1959. Montesquieu. Politique et histoire. Paris: PUF.

Althusser, L., 1976. Réponse à John Lewis. Paris: Maspero. 
Althusser, L., 1991. L'avenir dure longtemps. Paris: Stock Imec.

Althusser, L., 1994. Le courant souterrain du matérialisme de la rencontre. Dans: Ecrits philosophiques et politiques 1. Paris: IMEC STOCK, pp. 539-579.

Althusser, L., 1994. Marx dans ses limites. Dans: Ecrits philosophiques et politique 1. Paris: IMEC Stock, pp. 357-524.

Althusser, L., 1997. Du « Capital » à la philosophie de Marx. Dans: Lire le Capital. Paris: PUF, pp. 1-79.

Althusser, L., 1997. L'objet du Capital. Dans: Lire le Capital. Paris: PUF, pp. 245-418.

Althusser, L., 1998. La solitude de Machiavel. Paris: PUF.

Althusser, L., 1998. Sur le Contrat social. Dans: La solitude de Machiavel. Paris: PUF, pp. 59102.

Althusser, L., 2005. Pour Marx. Paris: La Découverte.

Althusser, L., 2009. Machiavel et nous. 2 éd. Paris: Thailander.

Althusser, L., 2011. Sur la reproduction. Paris: PUF.

Althusser, L., 2015. Être marxistes en philosophie. Paris: PUF.

Althusser, L., 2016. Les vaches noires. Paris: PUF.

Althusser, L., 2018. Ecrits sur l'histoire. Paris: PUF.

Balibar, E., 1991. Adieu. Dans: Ecrits pour Althusser. Paris: La Découverte, pp. 119-123.

Balibar, E., 1991. Ecrits pour Althusser. Paris: La Découverte.

Balibar, E., 1991. Tais-toi Althusser. Dans: Ecrits sur Athusser. Paris: La Découverte, pp. 59-89.

Balibar, E., 1993. L'objet d'Althusser. Dans: Politique et philosophie dans l'oeuvre d'Althusser. Paris: PUF (ed Kindle), pp. 1384-2005.

Derrida, J., 1990. Du droit à la philosophie. Paris: Galilée.

Duso, G., 2013. Libertà e costituzione in Hegel. Milano: FrancoAngeli.

Koselleck, R., 1972. Einleitung. Dans: Geschichtliche Grundbegriffe.. Stuttgart: Klett-, pp. XIIXXVIII.

Marx, K., 1969. Le Capital Livre 1, T. 1. Paris: Editions sociales.

Marx, K., 1972. Contribution à la crtiique de l'économie politique (1859). Paris: Editions sciences sociales. 
Marx, K., 1980. Manuscrits de 1857-1858 (Grundrisse) Tome 1. 1 éd. Paris: Editions sociales.

Marx, K. \& Lefebvre, J.-P., 1993. Le Capital. 2 éd. Paris: PUF.

Marx, K. \& Roy, J., 1969. Le Capital, Livre premier. Paris: éditions sciences sociales.

Montag, W., 2012. Le Lénine d'Althusser. Période. Revue en Ligne.

Moreau, P.-F., 1997. Althusser et Spinoza. Dans: Althusser philosophe. Paris: PUF , pp. 75-86.

Moreau, P.-F., 2001. Althusser et Machiavel. Dans: L'enjeu Machiavel. Paris: PUF, pp. 141-148.

Rancière, J., 1987. Le maître ignorant. Paris: Fayard.

Rancière, J., 1993. La scène du texte. Dans: Politique et histoire dans l'oeuvre d'Althusser. Paris: PUF - Kindle.

Rancière, J., 2011. La leçon d'Althusser. Paris: La Fabrique.

Rousseau, J.-J., 1964. Discours sur les Sciences et sur les Arts. Dans: Ouevres Complètes III. Paris: Gallimard, pp. 3-110.

Vincent, J.-M., 1991. La lecture symtomale chez Althusser. Dans: Poltique et histoire dans l'oeuvre d'Althusser. Paris: PUF, pp. 1128-1379.

REÇU: $12 / 04 / 2019$ APPROUVÉ: 02/02/2020

RECEIVED: 04/12/2019

APPROVED: 02/02/2020 